\title{
Infrared suppression of the Coulomb gauge gluon propagator in SU(3) Yang-Mills theory
}

\author{
Yoshiyuki Nakagawa* \\ Research Center for Nuclear Physics, Osaka University \\ Ibarakisi, Osaka 567-0044, Japan \\ E-mail: nkgwercnp.osaka-u.ac.jp
}

\section{Atsushi Nakamura}

Research Institute for Information Science and Education, Hiroshima University

Higashi-Hiroshima 739-8521, Japan

E-mail: nakamura@riise.hiroshima-u.ac.jp

\section{Takuya Saito}

Integrated Information Center, Kochi University

Kochi, 780-8520, Japan

E-mail: tsaitoulkochi-u.ac.jp

\section{Hiroshi Toki}

Research Center for Nuclear Physics, Osaka University

Ibarakisi, Osaka 567-0044, Japan

E-mail: toki@rcnp.osaka-u.ac.jp

\begin{abstract}
We calculate the equal-time transverse gluon propagator in Coulomb gauge QCD using a SU(3) quenched lattice gauge simulation on large lattices, up to $11^{4}\left[\mathrm{fm}^{4}\right]$. We find that the equal-time gluon propagator shows scaling violation; namely, the data for different lattice spacings do not fall on top of one curve. This problem is cured by discarding data at large momenta, which suffer from discretization errors. In the infrared region, the transverse gluon propagator is strongly suppressed and shows a turnover at about $500[\mathrm{MeV}]$. Fitting the power law ansatz to the data at small momenta predicts the vanishing gluon propagator at zero momentum, indicating the confinement of gluons.
\end{abstract}

8th Conference Quark Confinement and the Hadron Spectrum September 1-6 2008

Mainz, Germany

${ }^{*}$ Speaker. 


\section{Introduction}

Among several scenarios of color confinement proposed since the discovery of $\mathrm{QCD}$, Coulomb gauge QCD has recently been received much attention along lattice QCD simulations and a variational approach. Coulomb gauge is a physical gauge in the sense that unphysical degrees of freedom, such as longitudinal component of gluons, are integrated out and the color Gauss' law is formally solved. As a result, an instantaneous interaction shows up in the Hamiltonian in Coulomb gauge QCD, which plays a central role in the confinement mechanism in Coulomb gauge. In the Gribov-Zwanziger scenario, the path integral is dominated by the configurations near the Gribov horizon where the lowest eigenvalue of the Faddeev-Popov (FP) operator vanishes, and the eigenvalue distribution of the FP operator gets concentrated near the vanishing eigenvalue compared to that in the abelian gauge theory [1]. Such an enhancement has been observed by the lattice simulations $[2,3]$. Accordingly, the color-Coulomb instantaneous interaction is strongly enhanced and provides a confining force between color charges. Lattice QCD simulations have showed that the instantaneous color-Coulomb potential rises linearly at large distances and it is stronger than the static potential $[4,5,6,7]$. This is an expected result from the Zwanziger's inequality [8]. On the other hand, the color-Coulomb potential has been evaluated by inverting the FP matrix, and it has been shown that the color-Coulomb string tension almost saturates the Wilson string tension [9].

In the Gribov-Zwanziger scenario, the would-be physical gluon propagator is expected to be suppressed in the infrared (IR) region due to the proximity of the Gribov horizon in the IR direction [10]. In this study, we calculate the equal-time transverse gluon propagator,

$$
D_{\mu \nu}^{a b}(\vec{x}-\vec{y})=\left\langle A_{\mu}^{a}(\vec{x}) A_{\nu}^{b}(\vec{y})\right\rangle=D_{\mu \nu}^{a b}(\vec{x}-\vec{y}),
$$

in the momentum space,

$$
D_{i j}^{a b}(\vec{p})=\delta^{a b}\left(\delta_{i j}-\frac{p_{i} p_{j}}{\vec{p}^{2}}\right) D^{\mathrm{tr}}(|\vec{p}|) .
$$

using SU(3) quenched lattice QCD simulations. The lattice configurations are generated by the heat-bath Monte Carlo technique with the Wilson plaquette action. In these simulations we adopt the iterative method with the Fourier acceleration to fix a gauge, and the gauge fixing is stopped if $\left(\partial_{i} A_{i}\right)^{2}<10^{-14}$ at each time slice. The details of the simulations will be published elsewhere.

\section{Equal-time transverse gluon propagator}

The equal-time transverse gluon propagator at $\beta=5.7$ and 6.0 is drawn in the left panel of Fig. 1. The cone cut and the further cut are applied and the propagator is normalized such that $D^{t r}(|\vec{p}|=2[\mathrm{GeV}])=1$. The scale is set by using the Necco-Sommer scaling relation [11]. We observe that the gluon propagator has a maximum at $p=0.4 \sim 0.5[\mathrm{GeV}]$ irrespective of the lattice coupling and it decreases with decreasing the momentum in the IR region. This is the striking feature of the gluon propagator. The equal-time propagator is defined as the energy integral of the 4-dimensional propagator,

$$
D^{e q}(|\vec{p}|)=\int \frac{d p_{4}}{2 \pi} D\left(\vec{p}, p_{4}\right),
$$

and it can be interpreted as the inverse of the energy dispersion relation. Thus, the propagator at vanishing momentum corresponds to the inverse of the effective mass of the gluon. The IR 
suppression of the equal-time transverse gluon propagator implies that the gluons have momentum dependent effective mass $M(\vec{p})$ and it diverges in the IR limit, $\lim _{\vec{p} \rightarrow 0} M(\vec{p})=\infty$, indicating the confinement of gluons.
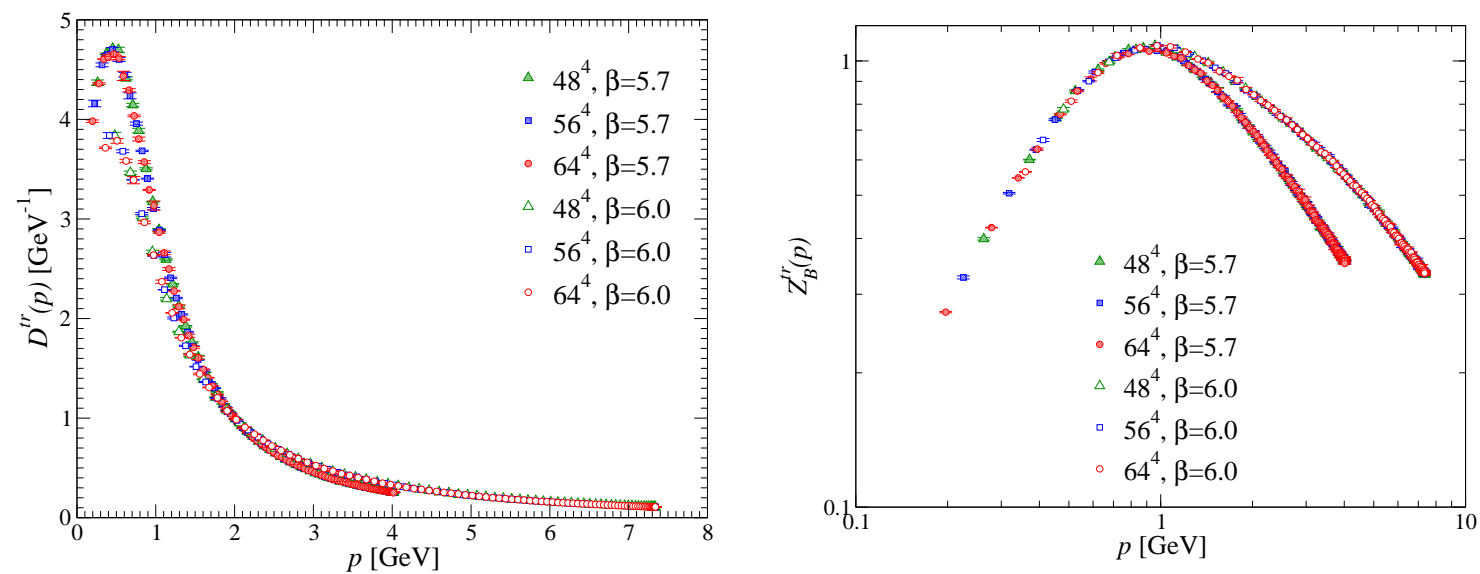

Figure 1: (Left) The equal-time transverse gluon propagator in physical units at $\beta=5.7$ and $\beta=6.0$. Data are converted from lattice units to physical units by adopting the Necco-Sommer scaling relation. The propagator is renormalized at $2[\mathrm{GeV}]$. (Right) The dressing function of the unrenormalized equal-time transverse gluon propagator, $Z_{B}^{t r}(\vec{p})=|\vec{p}| D_{B}^{t r}(\vec{p})$, at different lattice couplings is plotted in physical units.

The left panel of Fig. 1 also illustrates that the equal-time transverse gluon propagator shows scaling violation; namely, the data points at different lattice couplings cross at the renormalization point $|\vec{p}|=2[\mathrm{GeV}]$ and do not not fall on top of the same curve $[12,13,14]$. Taking a closer look at the raw results of the numerical simulations gives us a clue to cure scaling violation. The right panel of Fig. 1 shows the dressing function of the unrenormalized equal-time gluon propagator in physical units. We observe that the dressing function at different lattice couplings shows completely different behavior at high momenta. On the other hand, the momentum dependence of the dressing function is quite similar in the small momentum region. This implies that the scaling violation can be cured by restricting the momentum to

$$
\left|p_{\mu} a\right| \leq \alpha<2,
$$

and discarding data at large momenta where data suffer from strong discretization errors. In order to find a reasonable value for $\alpha$ which guarantees the scaling behavior of the transverse gluon propagator, we adopt a matching procedure described in [15].

\section{Result of the matching analysis and the IR behavior}

We cut data at large momenta and apply the matching procedure to the transverse gluon propagator. We refer to $[15,16]$ for the details of the matching analysis. We performed the matching between data at $\beta=6.0$ and $\beta=5.7$. The ratios of the lattice spacing and the renormalization constant obtained by the matching are given in Table 1. Although $\chi^{2} / n d f$ is relatively large for $\alpha=0.7$, it takes smaller values for smaller $\alpha$. For $\alpha \leq 0.6, \chi^{2} / n d f$ takes acceptable values, and $R_{a}$ and $R_{Z}$ are stable against the change of $\alpha$. The equal-time transverse gluon propagator with the 


\begin{tabular}{cccc}
\hline \hline$\alpha$ & $R_{a}$ & $R_{Z}$ & $\chi^{2} / n d f$ \\
\hline 0.7 & $1.73_{2}^{1}$ & $0.967_{4}^{3}$ & 9.72 \\
0.6 & $1.74_{2}^{2}$ & $0.973_{5}^{5}$ & 2.16 \\
0.5 & $1.74_{2}^{2}$ & $0.974_{7}^{6}$ & 2.72 \\
0.4 & $1.74_{2}^{2}$ & $0.973_{10}^{10}$ & 2.11 \\
\hline \hline
\end{tabular}

Table 1: The matching results with the further cut $\left|p_{i} a\right| \leq \alpha . R_{a}=a(\beta=5.7) / a(\beta=6.0)$ is the ratio of the lattice spacings and $R_{Z}=Z(\beta=5.7) / Z(\beta=6.0)$ is that of the renormalization constants. The NeccoSommer scaling relation gives $R_{a}^{N S}=1.83$.

further cut $\alpha \leq 0.6$ is shown in Fig. 2. We observe that the data points for different lattice couplings nicely fall on the same curve. This implies that scaling behavior of the equal-time gluon propagator is recovered by cutting the data points at large momenta, which suffer from the discretization effects.

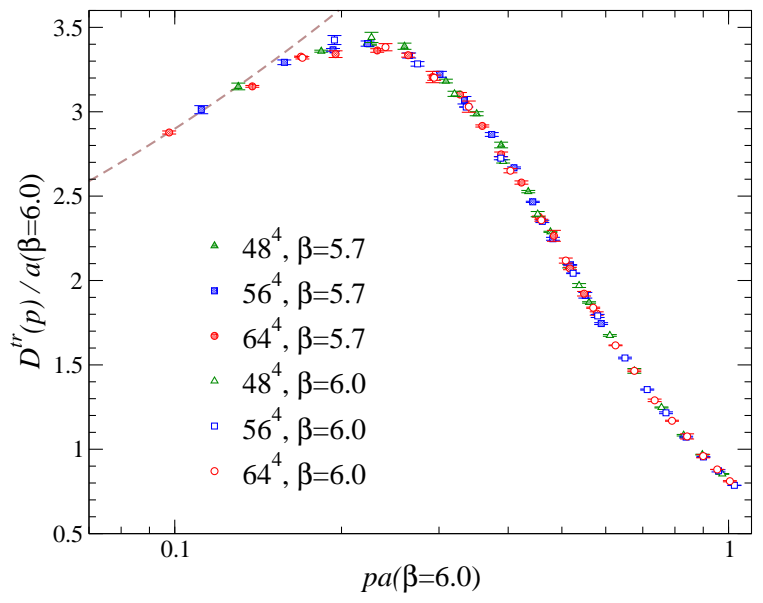

Figure 2: The matched gluon propagator with the further cut $\left|p_{i} a\right| \leq 0.6$. The 3-dimensional momentum and the propagator are expressed in units of the lattice spacing at $\beta=6.0$. The dashed curve represents the fitting function corresponding to the fitting range $0 \leq\left|\vec{p}_{\max }\right| a(\beta=6.0) \leq 0.135$.

In order to explore the IR behavior of the equal-time transverse gluon propagator, we make the power law ansatz,

$$
D^{t r}(|\vec{p}|)=d_{1}|\vec{p}|^{\gamma_{g l}^{I R}}
$$

in the IR region. The fitted parameters are listed in Table 2. The fitting function corresponding to the smallest fitting range is plotted in Fig. 2. Although the fitting becomes worse and the IR exponent $\gamma_{g l}^{I R}$ gets small as the maximum momentum of the fitting range increases, $\gamma_{g l}^{I R}$ takes positive value in all cases. That is, our result of the IR fitting predicts the vanishing transverse gluon propagator at zero momentum.

\section{Summary and conclusion}

We calculate the equal-time transverse gluon propagator on large lattices, up to $11^{4}\left[\mathrm{fm}^{4}\right]$. 


\begin{tabular}{cccc}
\hline$\left|\vec{p}_{\max }\right| a(\beta=6.0)$ & $d_{1}$ & $\gamma_{g l}^{I R}$ & $\chi^{2} / n d f$ \\
\hline 0.135 & $6.02(32)$ & $0.318(24)$ & 0.20 \\
0.140 & $5.29(11)$ & $0.261(10)$ & 3.49 \\
0.160 & $5.38(10)$ & $0.269(9)$ & 3.33 \\
0.170 & $5.29(5)$ & $0.261(5)$ & 2.64 \\
\hline
\end{tabular}

Table 2: The result of the IR power law fitting. $\left|\vec{p}_{\max }\right| a(\beta=6.0)$ represents the maximum momentum of the fitting range.

We find that the equal-time gluon propagator shows scaling violation. This problem is cured by discarding data at large momenta where the lattice data suffer from discretization errors. In the IR region, the transverse gluon propagator is strongly suppressed and shows the turnover at about 500 $[\mathrm{MeV}]$. Fitting the power law ansatz to the data at small momenta supports the vanishing gluon propagator at zero momentum, indicating the confinement of gluons.

\section{Acknowledgements}

The simulation was performed on NEC SX-8R at RCNP, Osaka University and NEC SX-9 at CMC, Osaka University. We appreciate the warm hospitality and support of the RCNP administrators.

\section{References}

[1] D. Zwanziger, Nucl. Phys. B412, 657 (1994).

[2] J. Greensite, S. Olejnik and D. Zwanziger, JHEP 05, 070 (2005).

[3] Y. Nakagawa, A. Nakamura, T. Saito and H. Toki, Phys. Rev. D75, 014508 (2007).

[4] J. Greensite and S. Olejnik, Phys. Rev. D67, 094503 (2003).

[5] A. Nakamura and T. Saito, Prog. Theor. Phys. 115, 189 (2006).

[6] Y. Nakagawa, A. Nakamura, T. Saito, H. Toki and D. Zwanziger, Phys. Rev. D73, 094504 (2006).

[7] Y. Nakagawa, A. Nakamura, T. Saito and H. Toki, Phys. Rev. D77, 034015 (2008).

[8] D. Zwanziger, Phys. Rev. Lett. 90, 102001 (2003).

[9] A. Voigt, E. M. Ilgenfritz, M. Müller-Preussker and A. Sternbeck, Phys. Rev. D78, 014501 (2008).

[10] D. Zwanziger, Nucl. Phys. B364, 127 (1991).

[11] S. Necco and R. Sommer, Nucl. Phys. B622, 328 (2002).

[12] Y. Nakagawa, A. Nakamura, T. Saito and H. Toki, PoS LAT2007, 319 (2007).

[13] A. Voigt, E.-M. Ilgenfritz, M. Müller-Preussker and A. Sternbeck, PoS LAT2007, 338 (2007).

[14] G. Burgio, M. Quandt and H. Reinhardt, 0807.3291.

[15] D. B. Leinweber, J. I. Skullerud, A. G. Williams and C. Parrinello, Phys. Rev. D 60, 094507 (1999).

[16] Y. Nakagawa, A. Voigt, E.-M. Ilgenfritz, M. Müller-Preussker, A. Nakamura, T. Saito, A. Sternbeck, and $\mathrm{H}$. Toki, in preparation. 